\title{
Direct evidence for continuous radar reflector originating from changes in crystal-orientation fabric
}

\author{
O. Eisen, I. Hamann, S. Kipfstuhl, D. Steinhage, and F. Wilhelms \\ Alfred-Wegener-Institut für Polar- und Meeresforschung, Bremerhaven, Germany
}

Received: 23 May 2007 - Published in The Cryosphere Discuss.: 15 June 2007

Revised: 5 September 2007 - Accepted: 1 October 2007 - Published: 19 October 2007

\begin{abstract}
The origin of a strong continuous radar reflector observed with airborne radio-echo sounding (RES) at the EPICA deep-drilling site in Dronning Maud Land, Antarctica, is identified as a transition in crystal fabric orientation from a vertical girdle to an increased single-pole orientation seen along the ice core. The reflector is observed with a $60 \mathrm{~ns}$ and $600 \mathrm{~ns}$ long pulse at a frequency of $150 \mathrm{MHz}$, spans one pulse length, is continuous over $5 \mathrm{~km}$, and occurs at a depth of about 2025-2045 $\mathrm{m}$ at the drill site. Changes in conductivity as reflector origin are excluded by investigating the icecore profile, synthetic RES data, and a RES profile with different electromagnetic polarisation azimuths. The reflector's magnitude shows maximum values for polarisation parallel to the nearby ice divide and disappears for polarisation perpendicular to it, identifying the orientation of the girdle to lie in the vertical plane parallel to the ice divide. Observations allow us to extrapolate the crystal orientation feature along the reflector in space, with implications for ice-sheet dynamics and modeling.
\end{abstract}

\section{Introduction}

Natural ice found in ice sheets and glaciers shows anisotropic behaviour in various physical parameters (Petrenko and Whitworth, 1999). A body of ice with anisotropic orientation of crystals responds differently to applied stresses compared with isotropic ice, for which ice crystals have a random orientation. For anisotropic ice, ice flow could be enhanced by about an order of magnitude if stresses act parallel to the crystals' basal planes, but flow could be reduced by an order of magnitude if stresses act perpendicular to the basal planes (Paterson, 1994). Consequently, the general flow of ice and the response of ice sheets to changing environmental condi-

Correspondence to: O. Eisen

(olaf.eisen@awi.de) tions depends on the degree of anisotropy within. The current demand for advanced modeling of ice sheets requires the incorporation of anisotropic properties of ice and the spatial distribution of these properties for unraveling ice sheet history and predicting the future development (ISMASS Committee, 2004). Various ways have been developed to treat anisotropic rheology, as, for instance, summarised by Marshall (2005). The correlation between fabric changes and climate transitions (e.g. Durand et al., 2007), moreover, emphasizes that valuable information is carried by vertical and lateral fabric distributions. However, information about the distribution of crystals within the ice, the crystal orientation fabric (COF), is mainly available from ice coring, and restricted, therefore, to point locations. No standard procedure exists to date to determine the spatial variation of $\mathrm{COF}$ within ice sheets on a routine basis. This is, however, a key to an improved understanding and modeling of ice-sheet behaviour. Here, we present direct evidence that radio-echo sounding (RES) provides a formidable tool to map strong fabric changes occurring over a few 10s of meters in the vertical, and, moreover, carries the potential to map the lateral distribution of COF continuously.

Since the beginning of operational applications of RES in glaciological research three processes were suggested to cause continuous internal reflections from within the ice column (Bogorodsky et al., 1985; Dowdeswell and Evans, 2004): changes in density (Robin et al., 1969), conductivity (Paren and Robin, 1975), and changes in COF (Harrison, 1973). Whereas the isochronous property of reflection horizons originating from changes in density and conductivity is nowadays routinely exploited for a multitude of purposes, the detection and interpretation of reflections stemming from changes in the COF is still in the early stages. Partial reflection of a propagating radar pulse occurs where either the real $\left(\varepsilon^{\prime}\right)$ or imaginary part $\left(\varepsilon^{\prime \prime}\right)$ of the complex dielectric constant $(\varepsilon)$ changes. The possibility to detect fabric changes with RES arises from the anisotropy of permittivity

Published by Copernicus Publications on behalf of the European Geosciences Union. 

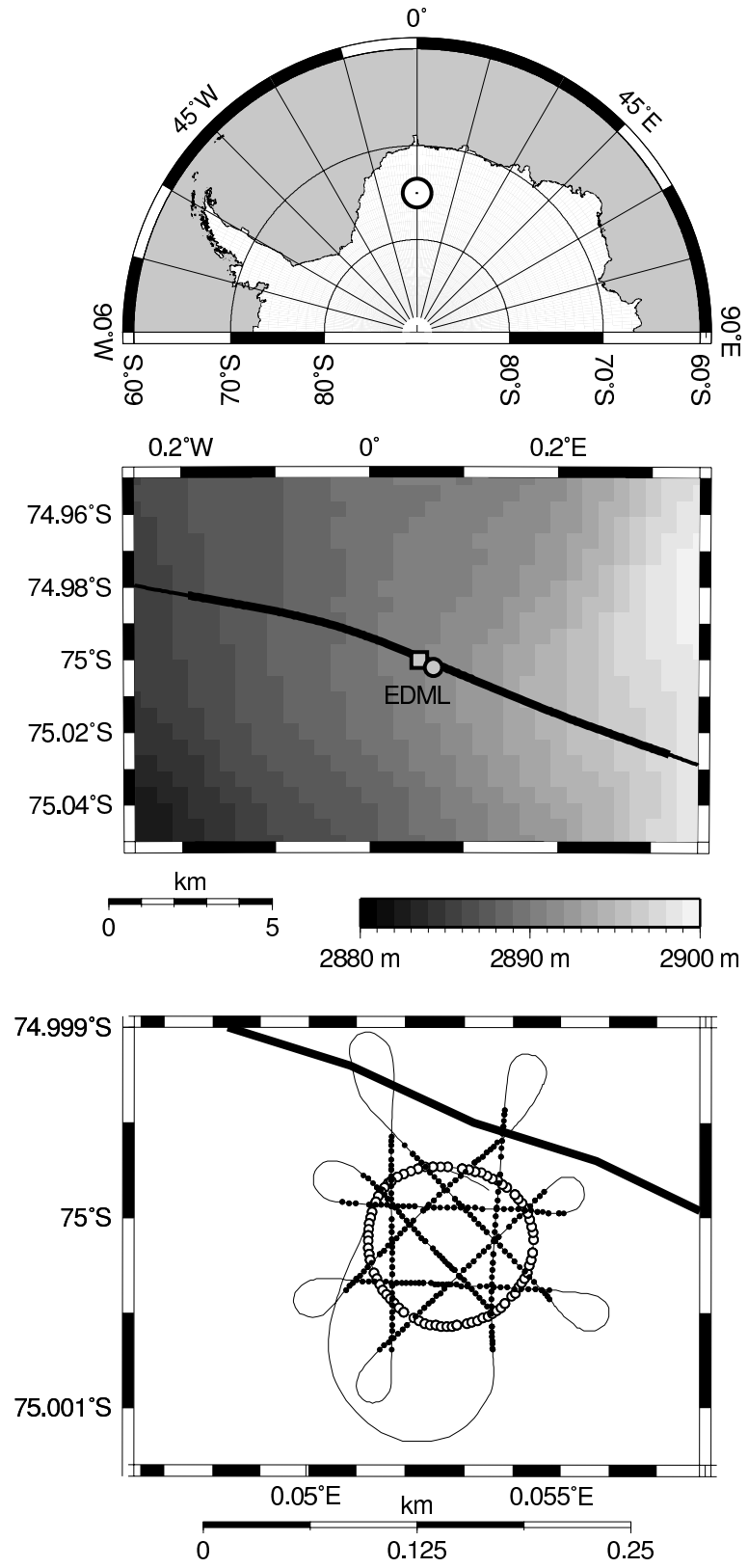

Fig. 1. Top: location of study area in Antarctica. Middle: location of EDML drill site (circle), RES flight line of profiles 022150/023150 (black line), and ground-based profile 033042 (square); bold black line: RES section shown in Fig. 2; grayscale: Radarsat Antarctic Mapping Project digital elevation model (Liu et al., 2001) on $400 \times 400 \mathrm{~m}^{2}$ raster (meter above sea level for WGS84 ellipsoid). Bottom: close-up of profile 033042 recorded with airplane on ground (thin line) and flight line of profiles $022150 / 023150$ (thick line). Traces of the circle segment of profile 033042 are indicated by open circles, traces belonging to linear segments are shown as black dots.

in a pure ice crystal. The latest compilation of dielectric properties of ice (Fujita et al., 2000) provides an anisotropy in $\varepsilon^{\prime}$ of $\Delta \varepsilon^{\prime}=\varepsilon_{\|}^{\prime}-\varepsilon_{\perp}^{\prime} \approx 0.035$, measured parallel and perpen- dicular to the crystal c-axis at $1 \mathrm{MHz}$ and $252 \mathrm{~K}$. This value corresponds to little more than roughly $1 \%$ of the permittivity of ice, which is in the range of 3.1-3.2. $\Delta \varepsilon^{\prime}$ is accurate to $\sim 0.007$, or $20 \%$ (Fujita et al., 2000, Fig. 3), and varies slightly with temperature and frequency, however insignificantly to be a concern in this study.

To discriminate reflections originating from changes in $\mathrm{COF}$ and conductivity, multi-frequency experiments can be utilized (Fujita et al., 1999). However, as ice is also a birefringent medium at radio frequencies (Hargreaves, 1978), multi-polarisation measurements are required to resolve ambiguities arising from anisotropic reflection and wave propagation (Doake et al., 2002; Fujita et al., 2003). With such experiments, Matsuoka et al. (2003) located high-scattering zones several hundred meters thick at depths around 1000$1500 \mathrm{~m}$ in East Antarctica. Based on the dependence of the reflection strength on the polarisation plane, the wide zones were interpreted as alternations in COF of adjacent ice layers (Matsuoka et al., 2004), induced by past and present ice flow. Fujita et al. (2006) determined the vertical evolution of the radar phase by combining ice-core profiles of COF with a matrix-model of radar-wave propagation at the sites where polarimetric radar studies were carried out. By comparison of COF data and model results with multi-polarisation/bifrequency RES point data, they were able to separate the general contribution of anisotropic reflection from $\mathrm{COF}$ and birefringence to the observed signals. However, previous studies did not provide COF profiles from ice cores for direct comparison with continuous internal reflections in RES data and reflectivity.

In this work we extend previous findings by comparing RES data with ice-core profiles of COF, permittivity, and conductivity. Moreover, we use synthetic RES data to analyse the origin of RES signals. This leads to the novel observation that a sharp, continuous internal reflection horizon, trackable over several kilometers, is caused by an abrupt transition in crystal orientation fabric occuring over just a few tens of meters. Our deduction from these comparisons is supported by the analysis of the azimuth-dependence of reflection magnitude of the reflector of interest.

\section{Data and methods}

Data used in the present study were acquired within the European Project for Ice Coring in Antarctica (EPICA) at the deep drill site in Dronning Maud Land, Antarctica (EDML). The drill site is located at $0.0684^{\circ} \mathrm{E}, 75.0025^{\circ} \mathrm{S}$ at $2891.7 \mathrm{~m}$ above sea level near a transient ice divide, characterised by along-flow compression and lateral extension, causing an overall vertical compression of the ice column (Wesche et al., 2007). 


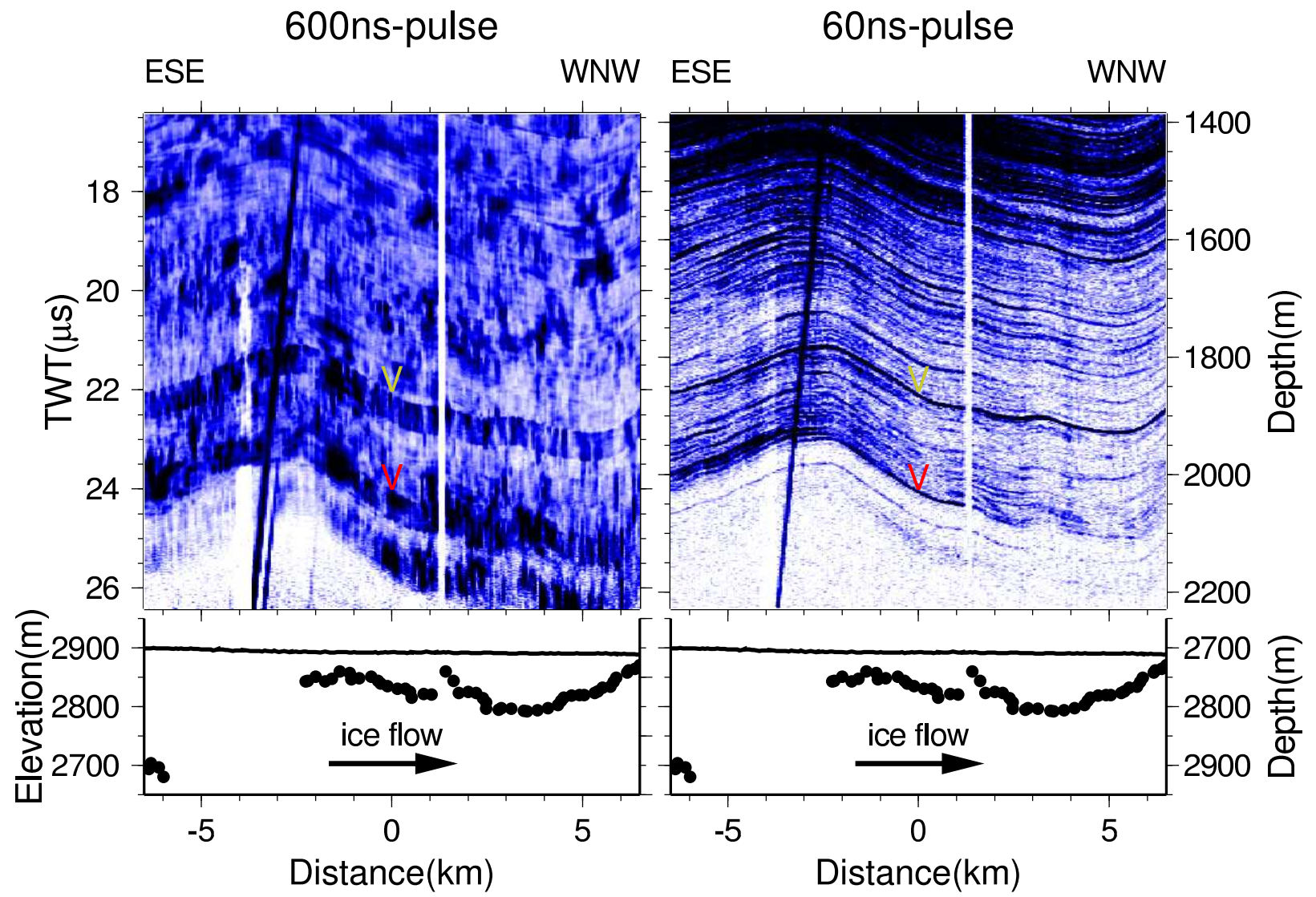

Fig. 2. Top: unfiltered 10 -fold stack (65-m trace spacing) RES profile recorded with the 600 -ns pulse (022150, left) and 60-ns pulse (023150, right); ordinates are the same for both panels: two-way travel time (TWT) on left ordinate is recording time corrected to the first break of surface reflection, right ordinate refers to depth below the surface for a mean wave speed of $168.7 \mathrm{~m}^{-1}$ from the surface to a 2100 - $\mathrm{m}$ depth; color code indicates signal magnitude (increasing white-blue-black); first breaks of internal reflectors of interest are marked by yellow and red "V" at $0 \mathrm{~km}$ (drill site) at travel times of 22 and $24 \mu \mathrm{s}$, respectively; dark diagonal line between -3.8 and $-2.3 \mathrm{~km}$ is a hyperbola leg, caused by reflections from Kohnen station (located at $0 \mathrm{~km}$ ); white vertical line at $1.3 \mathrm{~km}$ is caused by system dropout. Bottom: surface elevation above mean sea level (solid line, left axis) from airplane radar altimeter and depth of detected bedrock reflection below surface (black dots, right axes). The profile runs approximately parallel to a transient ice divide (ESE-WNW), arrow indicates ice flow from left to right.

\subsection{Radio-echo sounding (RES)}

We use data from the RES system on board the Dornier 228101 aircraft Polar2, operated by the Alfred Wegener Institute (AWI). The system, with an overall sensitivity of $190 \mathrm{~dB}$, generates $150-\mathrm{MHz}$ bursts of $60 \mathrm{~ns}$ and $600 \mathrm{~ns}$ in duration, which are transmitted alternatedly. The receiver module rectifies and logarithmically compresses the signals, stacks 200 consecutive signals after analog-to-digital conversion, and stores the stack as a single trace on tape at sample intervals of $13.33 \mathrm{~ns}$ over a time window of $50 \mu \mathrm{s}$. Data are normally acquired in-flight at an altitude of $450 \mathrm{~m}$ above ground at typical speeds of $65 \mathrm{~m} \mathrm{~s}^{-1}$ (130 knots), resulting in a trace spacing of the 200-fold stack of $6.5 \mathrm{~m}$ between equal pulses (Nixdorf et al., 1999). The airplane heading is equal to the polarisation of the electric field. Apart from static correction for the first break of the surface reflection, no additional filtering or gain control is applied to the RES data used here. Conversion from recorded travel time to depth domain is performed with the calibration for pure-ice permittivity derived by Eisen et al. (2006), with a mean wave speed of $168.7 \mathrm{~m} \mathrm{\mu s}^{-1}$ over the distance from the surface to $2100-\mathrm{m}$ depth.

We use two types of RES data sets here (Fig. 1): (i) airborne data from the season 2002/03, with profiles 022150 (600 ns pulse) and 023150 (60 ns pulse) running along the ice divide (ESE-WNW direction), passing the EDML drill site about $100 \mathrm{~m}$ to the NNE (Fig. 2); (ii) data from profile 033042 (60 ns pulse) recorded in season 2003/04 with the airplane slowly moving on the ground about $500 \mathrm{~m}$ downstream from the drill site, first in a circle and then crossing the circle in linear segments in eight different orientations (N, NE, E, SE, S, SW, W, NW). The airborne data enable 


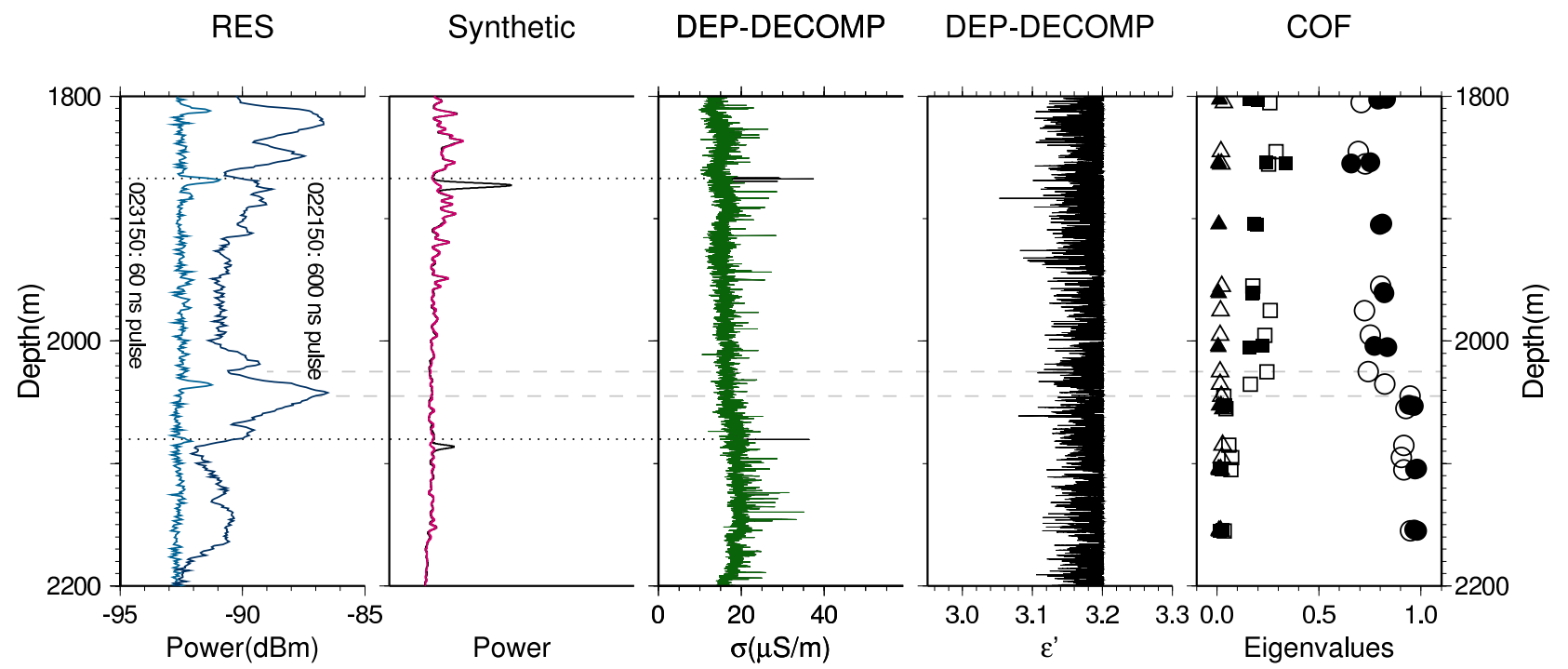

Fig. 3. Comparison of radar data and ice-core profiles at the EPICA-DML drill site. From left to right: RES signal power measured with two pulse widths for trace 4205 of profiles 022150 (600 ns pulse) and 023150 (60 ns pulse) with respect to $1 \mathrm{~mW}$ (dBm), representing a stack of 2000 recordings in total (200 pre-storage and 10 post-storage stacks) over a distance of 65 m; DEP-based synthetic RES trace for 60-ns pulse; DECOMP-corrected DEP conductivity $(\sigma)$; DECOMP-corrected DEP-permittivity ( $\varepsilon^{\prime}$, with the permittivity of pure ice as upper bound); eigenvalues $\left(\lambda_{1}\right.$ : triangle, $\lambda_{2}$ : square, $\lambda_{3}$ : circle) of crystal orientation tensor along horizontal (filled symbols) and vertical core samples (open symbols). The black synthetic trace is based on original conductivity data (black $\sigma$-curve); on top the red synthetic trace is plotted, based on the DEP data with conductivity peaks removed (green $\sigma$-curve); peaks originating from conductivity thus appear black, emphasized by dotted horizontal lines; dashed horizontal lines connect the depth of the strong RES reflector with transition depth of changes in $\mathrm{COF}$.

us to investigate the lateral extension of continuous internal reflections. For their subsequent analysis, we additionally stack 10 traces of each pulse width (resulting in a 65-m trace spacing in Fig. 2). The traces closest to the drill site are used later for comparison with ice-core data. They are referred to as the long and short pulse RES traces, respectively (Fig. 3). The ground-based data of profile 033042 provide insights into the dependence of reflected energy on polarisation azimuth. Along each of the eight linear segments of constant heading, we stack 70 consecutive traces to increase the signal-to-noise ratio. The data from the circle segment are used unstacked.

\subsection{Ice-core permittivity and conductivity profiles}

Dielectric profiling (DEP) at $250 \mathrm{kHz}$ and $\gamma$-attenuation profiling, simultaneously carried out in the field after core retrieval, provide dielectric properties and density along the EDML ice core (see Eisen et al., 2006, for details). The calibrated DEP record is corrected for variations in core diameter and temperature. Unreliable data in the vicinity of the core breaks are removed. Effects of density and conductivity mixed complex-valued permittivity in the two-phase system ice-air are taken into account by applying an extended volumetric mixing model (DECOMP). Thus, contributions from density and conductivity in the complex permittivity are separated (Wilhelms, 2005).

\subsection{Finite-difference forward modeling}

The synthetic trace, adapted from Eisen et al. (2006), simulates the 60-ns pulse (Fig. 3). It is calculated by a one-dimensional finite-difference time-domain model of Maxwell's curl equations. Ice-core profiles of permittivity and conductivity serve as model input, separated using an inverse application of DECOMP at $250 \mathrm{kHz}$, conductivity linearily scaled to $150 \mathrm{MHz}$, and merged again with forward application of DECOMP. Conductivity is provided in 0.02-m resolution. Due to the strong influence of the DEPmeasurement noise in permittivity on synthetic reflections, permittivity is smoothed over $20 \mathrm{~m}$ for forward modeling (Eisen et al., 2006). The synthetic trace thus only contains reflections which originate from changes in conductivity. Processing comprises a synchronization of the synthetic source signal to the RES first-break signal from the surface by a time shift of $+0.1 \mu \mathrm{s}$, application of a Hilbert magnitude transformation to obtain a signal envelope (i.e. mimic rectification), and a smoothing with a $0.1-\mu$ s Gaussian running-mean filter. Details are presented in Eisen et al. (2006)

\subsection{Determination of crystal-orientation fabric}

Fabric data of the EDML deep ice core were measured down to a 2560-m depth in a coldroom at a temperature of $-20^{\circ} \mathrm{C}$ in 2005 at AWI (Bremerhaven, Germany). Ice-core sections 
were drilled between 2001 and 2004 and have been stored at $-30^{\circ} \mathrm{C}$ after the transportation at $-25^{\circ} \mathrm{C}$ to AWI. Thin sections were prepared according to standard procedures using a microtome from horizontally $\left(0.5 \times 50 \times 50 \mathrm{~mm}^{-3}\right)$ and vertically $\left(0.5 \times 50 \times 100 \mathrm{~mm}^{-3}\right)$ cut samples. Orientations of caxes were derived using an automatic fabric analyzer system (Wilson et al., 2003), which enables complete measurement of these samples in 15 to $30 \mathrm{~min}$. Unfortunately, it is not yet possible to record the horizontal azimuth of the drilled core sections, so azimuth is not available for the thin sections. In addition to the Schmidt diagrams (mapping each measured crystal c-axis direction from a point on the hemisphere to a circular plane using an equal-area projection, Fig. 4), we present the data in terms of the eigenvalues $\lambda_{i}$ of the orientation tensor (Wallbrecher, 1979). The eigenvalues (Fig. 3) represent the components along the orthogonal coordinate system $\hat{e}_{i}$ of an ellipsoid, which best approaches the momentum of inertia of the c-axes distribution. Measurements of samples cut horizontally and vertically from the core are jointly displayed. From the distribution of all measurements along the whole core it is evident that the eigenvalues are accurate to about a value of \pm 0.1 . This is partly explained by the physical variations in the distribution of crystals in each sample, but also partly by local effects attributed to the immatureness of the system. Nevertheless, the eigenvalues are considered reliable within this accuracy.

\section{Results and discussion}

Drilling at EDML finished in January 2006, reaching bedrock at a logged ice thickness of $2774.15 \mathrm{~m}$. This corresponds well with the ice thickness determined by RES (Fig. 2). The RES profiles at both pulse widths show the common internal layering of ice sheets, which increasingly follows the bedrock topography at larger depth, especially evident for profile sections spanning several 10-100 km (not shown). A sudden decrease in reflected energy and the absence of any laterally continuous signals suggest that the echo-free zone is present in the lowest $500-800 \mathrm{~m}$ above bedrock, starting at about $2100 \mathrm{~m}$ at the drill site.

The $600 \mathrm{~ns}$ pulse spans some $50 \mathrm{~m}$ in ice and integrates over a much wider depth range than the short pulse, which covers only about $5 \mathrm{~m}$. As would be expected, fewer layers are therefore visible in the profile recorded with the $600 \mathrm{~ns}$ pulse than in the one recorded with the 60 ns pulse (Fig. 2). Looking at single traces, it is evident that several distinct peaks in the short-pulse trace have nevertheless counterparts in the long-pulse trace. This is clearly the case for strong reflections at depths of 1810 and $2035 \mathrm{~m}$, and less so at 1865 and $2080 \mathrm{~m}$ (Fig. 3). Dominant reflections of each pulse cover about one pulse length. The first break of the concurrent reflections appear at about the same depth, but the maximum power in the long pulse occurs about $10 \mathrm{~m}$ deeper than in the short pulse.

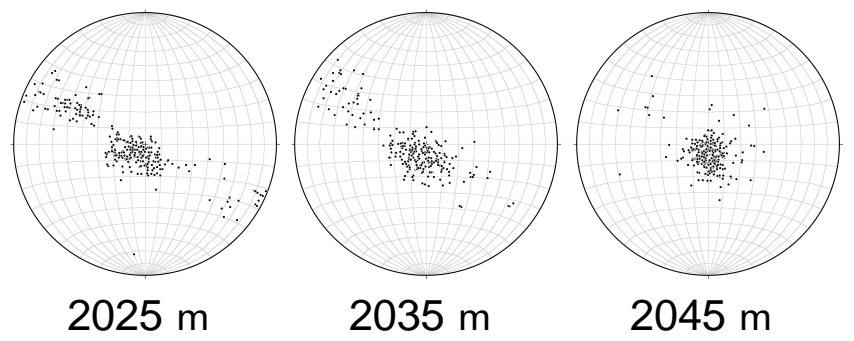

Fig. 4. Schmidt diagrams of vertical sections at three depths, showing the orientation of c-axes and the transition from vertical girdletype to increased vertical single-maximum fabric. The orientations have been rotated into the horizontal $x y$-plane.

The comparison between the short pulse trace and the synthetic trace carried out by Eisen et al. (2006) demonstrates that a number of internal layers can be reproduced by forward modeling based on a conductivity profile. Removing dominant peaks by interpolation in the underlying conductivity profile causes a disappearance of the related reflections in the synthetic trace, as indicated for two cases at 1865- and 2080-m depth in Fig. 3 by the dotted line and the black peaks of the synthetic radargram. This demonstrates that these reflections originate from narrow peaks in conductivity, all less than about $0.5 \mathrm{~m}$ wide (Eisen et al., 2006). Interestingly, the strong signal in the short pulse at $2035 \mathrm{~m}$ is not reproduced in the synthetic data. The origin of this reflection and its characteritic properties will be investigated in detail in the remaining part of the paper.

\subsection{RES vs. ice-core data}

In comparison to the reflection at $1865 \mathrm{~m}$ of the short pulse, which is caused by a peak with a maximum conductivity of $38 \mu \mathrm{S} \mathrm{m}^{-1}$ on a background conductivity of $15 \mu \mathrm{S} \mathrm{m}^{-1}$, one would expect a conductivity peak of comparable size also at $2035 \mathrm{~m}$. However, the conductivity profile merely displays a peak of $24 \mu \mathrm{S} \mathrm{m}^{-1}$ on a background of $17 \mu \mathrm{S} \mathrm{m}^{-1}$ at this depth. Peaks of this size appear regularly in the conductivity profile, without causing strong reflections, neither in the short pulse RES data nor in the synthetic trace. Missing data, rejected in the DEP quality check, could mask a prominent conductivity peak. Using $0.5 \mathrm{~m}$ as the upper limit for the width of strong conductivity peaks responsible for reflections, as identified by Eisen et al. (2006), the closest sections of data gaps $>0.5 \mathrm{~m}$ are located at 2026.262027.32, 2028.29-2028.81, 2038.90-2039.93, and 2040.03$2040.65 \mathrm{~m}$. Each of these gaps is further away from the reflector's depth of origin than its depth accuracy of less than $1 \mathrm{~m}$ (Eisen et al., 2006). We therefore exclude the possibility that a single peak in conductivity causes the reflection in the short pulse trace at $2035 \mathrm{~m}$.

Another possibility for the origin of the reflection at 2035$m$ depth would be that interference of partial reflections at 

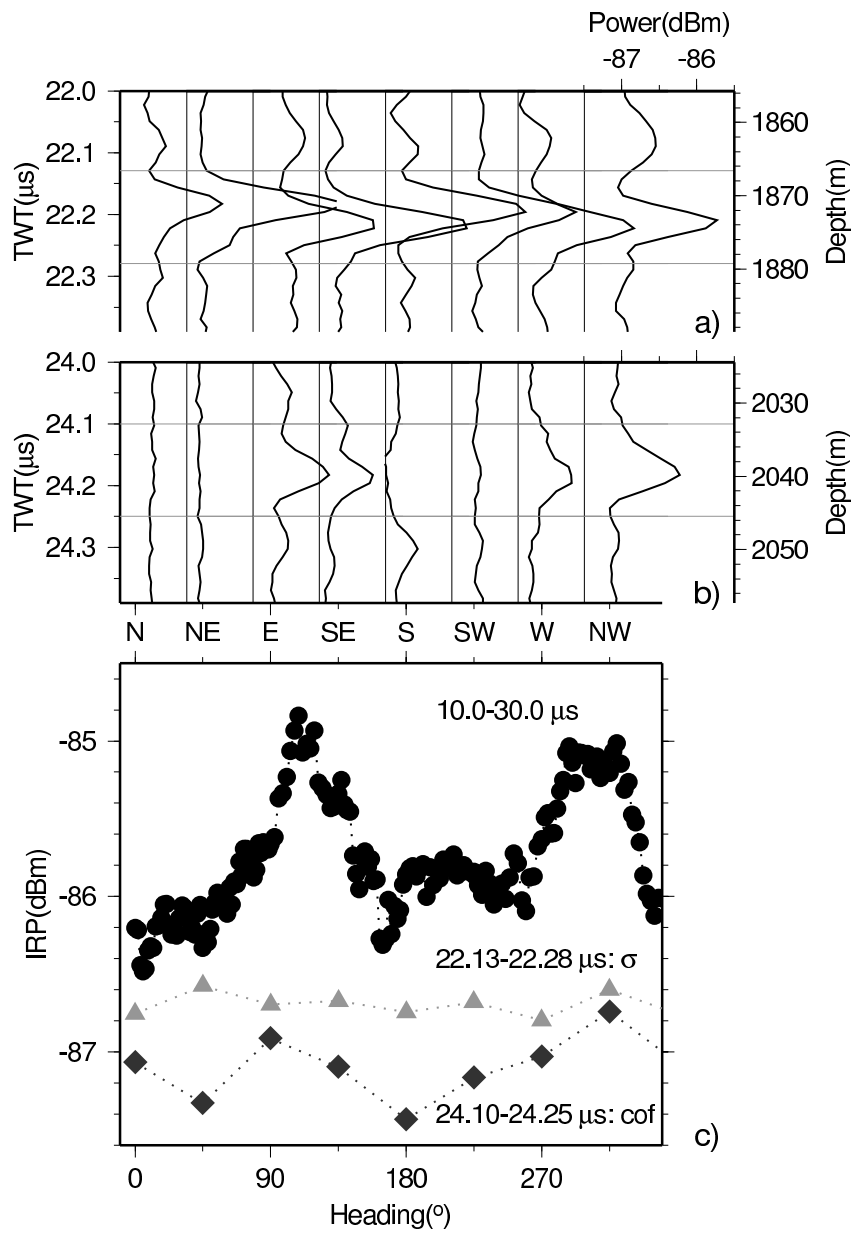

Fig. 5. Comparison of azimuth dependence for two reflectors originating from changes in (a) conductivity and (b) COF for eight airplane headings, which are equal to electric polarisation. Each trace in (a) and (b) is a stack of 70 traces along one of eight linear segements of profile 033042 (Fig. 1), recorded with the 60-ns pulse; power scale given for heading NW in a) applies for all traces; vertical baselines in (a) and (b) correspond to $-87.5 \mathrm{dBm}$; horizontal lines bound time windows used for calculation of internal reflection power (IRP) displayed in (c). (c) IRP as a function of azimuth and time window, calculated as the power average per sample over the respective time window. Triangles and diamonds: IRP over 150-ns time window (12 samples) of traces shown in (a) and (b), respectively; black circles: IRP over 10-30 $\mu$ s time window for unstacked traces of circle segment of profile. The ice divide runs along the ESE-WNW direction.

several conductivity peaks within one pulse length occurs. However, referring to the reflection's counterpart in the longpulse data, it would be surprising that a series of small conductivity peaks of just $5-10 \mu \mathrm{S} \mathrm{m}^{-1}$ above a background of $15-20 \mu \mathrm{S} \mathrm{m}^{-1}$ would allow for coherent interference in both pulse lengths to produce the dominant reflections being one pulse width long. We therefore also exclude the possibility that the reflections at $2035 \mathrm{~m}$ in both RES traces are caused by changes in conductivity, and thus the imaginary part $\varepsilon^{\prime \prime}$ of the dielectric constant.

The only other possibility for reflector origin is the real part $\varepsilon^{\prime}$. At these depths, changes in density are not present, as air bubbles already turned into air hydrates. As these have a very low volume density (Matsuoka et al., 2004), they hardly influence the bulk permittivity. One would consequently expect a profile of basically constant permittivity. The noise seen in the permittivity profile of about 0.05 on the sub-meter scale (Fig. 3) is attributed to measurement noise of the DEP bench. We therefore cannot use the permittivity profile to determine the reflection origin.

The remaining possibility to cause a change in $\varepsilon^{\prime}$ are changes in COF. The Schmidt diagrams at 2025-2045-m depth in Fig. 4 show a transition from a vertical girdle-type to a single-maximum fabric with c-axes clearly concentrated around the vertical. The eigenvalues of the distribution of c-axes (Fig. 3) show this transition very clearly in the two largest components, $\lambda_{2}$ and $\lambda_{3}$, which change by $\sim 20 \%$ over $20 \mathrm{~m}$. A change from a vertical girdle-type fabric to a vertically concentrated fabric as reflector origin should result in an anisotropic response in the RES signals as a function of polarisation. This will be investigated next.

\subsection{Analysis of RES polarisation azimuth}

The RES data of profile 033042 were recorded with different airplane headings, and thus polarisation azimuths (Fig. 1). This data set provides an independent means to determine the origin of the internal reflections and enables us to fix the azimuth ambiguities of the COF measurements along the core.

For different time windows we calculate the internal reflection power (IRP, compare Gades et al., 2000), defined as the power average per sample (in $\mathrm{dBm}$ ) within the time window. The azimuth dependence of the reflected energy from individual reflectors (i.e. for short time windows) is difficult to analyse for the circle segment, as the signal-to-noise ratio is very low and stacking is not feasible because of the different azimuths. However, IRP over the time window 10-30 $\mu$ s indicates that for the heading $\mathrm{E}$ to $\mathrm{SE}$ and $\mathrm{W}$ to NW the IRP is significantly higher than for other headings (Fig. 5). The maxima lie in the vertical plane along the ice divide (ESEWNW).

Our explanation is as follows: c-axes orientations of all types (single maximum, girdle, ...) show small statistical variations around a mean distribution with depth. Thus, the dielectric properties also vary slightly with depth, causing some incoherent backscatter of a propagating radar signal. These variations have the largest effect on the radar backscatter if they occur in the vertical plane parallel to radar polarisation. At the present location, dominated by a girdle-type fabric between 450 and 2025-m depth, polarisation perpendicular to the ice divide results in an overall lower level of backscatter, whereas for polarisation parallel to the ice divide the level of backscatter is highest. We interpret this as 
an indication that the girdle fabric lies in the vertical plane parallel to the ice divide, and that variations in the girdle fabric cause the higher backscatter in that plane. For later analysis, we define this as the $y z$-plane of an orthogonal coordinate system, with the horizontal coordinates $x$ and $y$ as the acrossflow (NNE) and the along-flow (WNW) directions, respectively, and the vertical coordinate $z$.

We next look at the variation of reflected energy as a function of azimuths of the stacked linear segments. For the reflector originating at $1865-\mathrm{m}$ depth at the drill site, both the display of the raw traces (Fig. 5a), as well as the IRP calculated over a 150-ns window containing the reflection (Fig. 5c) do not show any clear systematic variation in backscatter with azimuth. This isotropic pattern is expected for reflections stemming from changes in conductivity, as from the peak present at $1865 \mathrm{~m}$ in the ice-core data. At the depth of the change in COF at about $2035 \mathrm{~m}$ at the drill site, a reflection is visible for headings $\mathrm{E}$ and $\mathrm{SE}$, and in the opposite directions $\mathrm{W}$ and NW. Along the other headings no reflection is visible (Fig. 5b). The IRP, again calculated for a 150-ns time window at these depths, likewise shows maxima for the headings which also show a reflection (Fig. 5c). We interpret this to be caused by the change in COF from the anisotropic distribution of c-axes of the girdle-type fabric, which has an isotropic distribution in the horizontal plane, to the vertically concentrated fabric. If the electric polarisation is parallel to the plane in which c-axes change, i.e. parallel to the ice divide in the $y z$-plane, permittivity changes as well, and a reflection occurs. If, in contrast, the electric polarisation is perpendicular to the plane containing the c-axes, then any changes in COF in the $y z$-plane will not influence the radar wave. We therefore take the azimuth-dependence of reflectivity as an independent confirmation that the reflector at $2035 \mathrm{~m}$ indeed originates from the changes in COF observed in the ice core.

\subsection{Reflectivity from ice-core $\mathrm{COF}$}

The distribution of c-axes allows us to calculate the permittivity components. Following Fujita et al. (2006), the effective permittivity in the direction of the ellipsoid's principle components $\hat{e}_{i}$ can be determined from the eigenvalue $\lambda_{i}$ by $\varepsilon_{i}^{\prime}=\varepsilon_{\perp}^{\prime}+\Delta \varepsilon^{\prime} \lambda_{i}$. In the present case, the principle component $\hat{e}_{3}$ is very close to the vertical below 1700-m depth (Fig. 4), and $\hat{e}_{1,2}$ lie in the horizontal $x y$-plane. Although borehole logging indicated that the core axis has a possible deviation of $1-2^{\circ}$ from the vertical, we consider this negligible for the following analysis, as the accuracy of the fabric analyzer is on the same order of magnitude. This is moreover justified by the width of the distribution of c-axes of some tens of degrees, even for the vertically highly concentrated fabric. The results above with azimuth dependence of backscatter from the circle profile indicate that $\hat{e}_{1}$ corresponds to the $x$ direction, and $\hat{e}_{2}$ to the $y$-direction. The principle compo- nents $\hat{e}_{i}$ are therefore approximately aligned with the orthogonal coordinates $x, y, z$.

The change in COF between depths $z_{1}=2025$ and $z_{2}=2045 \mathrm{~m}$ results in a change in the permittivity components $\delta \varepsilon_{i}^{\prime}=\left.\varepsilon_{i}^{\prime}\right|_{z_{1}}-\left.\varepsilon_{i}^{\prime}\right|_{z_{2}}=\Delta \varepsilon^{\prime}\left(\left.\lambda_{i}\right|_{z_{1}}-\left.\lambda_{i}\right|_{z_{2}}\right)$. Using the eigenvalues determined in the vertical sections yields $\delta\left(\varepsilon_{x}^{\prime}, \varepsilon_{y}^{\prime}, \varepsilon_{z}^{\prime}\right)$ $=\Delta \varepsilon^{\prime}(0.6,21.1,-20.5) 10^{-2}=(0.2,7.4,-7.2) 10^{-3}$. This change occurs over $20 \mathrm{~m}$, well within the width covered by the long pulse. The radar wave propagates in the vertical direction, so only $\delta \varepsilon_{x, y}^{\prime}$ can cause a reflection. Using the approximation from Paren (1981) to determine the power reflection coefficient for a two-layer interface with differing permittivities, $|R|=\left(\delta \varepsilon^{\prime} /(4 \varepsilon)\right)^{2}$, the change in $\delta \varepsilon_{x}^{\prime}$ corresponds to a reflection coefficient $R_{x}=-96.1 \mathrm{~dB}$, and the change $\delta \varepsilon_{y}^{\prime}$ yields $R_{y}=-64.6 \mathrm{~dB}$. The measurement error of $20 \%$ in $\Delta \varepsilon^{\prime}=0.035$ results in an uncertainty of less than $1 \mathrm{~dB}$ of $R_{x}$ and $R_{y}$. Whereas $R_{x}$ is very small, likely too small to result in a sufficiently large reflection, $R_{y}$ seems to be large enough to detect a related reflection at the surface. These reflection coefficients explain the above observation that the reflection at $2035 \mathrm{~m}$ only appears for polarisation in $y$-direction (parallel to the ice divide, Fig. 5b), but not in the $x$-direction (perpendicular to the ice divide).

The nearby reflections at $2080 \mathrm{~m}$, appearing for both pulse lengths, provide a qualitative plausibility check for the hypothesis that the change in COF in the $y z$-plane is indeed strong enough to cause reflections. As shown by the synthetic trace, the reflections' origin is the conductivity peak at $2080 \mathrm{~m}$ with a height of $37 \mu \mathrm{S} \mathrm{m}^{-1}$. The reflection coefficient corresponding to this conductivity peak (Paren, 1981) results as $-75.7 \mathrm{~dB}$ at $150 \mathrm{MHz}$, only a few $\mathrm{dB}$ smaller than the reflection coefficients $R_{y}$ resulting from the change in COF. The smaller reflection coefficient is consistent with the fact that the reflections' maximum peak power at $2080 \mathrm{~m}$ for both pulses are smaller than those at $2035 \mathrm{~m}$. We can therefore indeed attribute the origin of the observed reflections at $2035 \mathrm{~m}$ to the change in COF, which is strong enough and occurs over a short enough distance to cause the strong reflections in the short and long pulse data.

\subsection{Distribution of reflector from COF}

Laterally, the reflector from COF (red "V" in Fig. 2) is quasiparallel to other internal layers, e.g. the reflectors from conductivity at a depth of $1865 \mathrm{~m}$ (yellow "V") and $2080 \mathrm{~m}$ at the drill site, but displays stronger variations in reflection magnitude. Although the 600-ns pulse radargram contains more noise (in terms of clutter or speckle) than the 60-ns data, the reflector from conductivity at $1865 \mathrm{~m}$ is clearly dominant. Both reflectors from conductivity can clearly be tracked over the whole section of the 60-ns pulse in Fig. 2. The reflector from COF seems to be continuous over the whole section in the 600-ns data as well, although of very low quality. In contrast, it can only be tracked between $-3 \mathrm{~km}$ and $2.5 \mathrm{~km}$ in the 60 -ns data. It is very strong on the downstream 
(right) side of the anticline between -3 and $1.5 \mathrm{~km}$. Further upstream (to the left) from the anticline, it fades away, with several strong reflectors appearing within $20 \mathrm{~m}$ above it. Downstream from the drill site, it starts to fade away at $1.5 \mathrm{~km}$, and is interrupted and lost in the syncline starting around $3 \mathrm{~km}$. The different characteristics of the $60 \mathrm{~ns}$ and $600 \mathrm{~ns}$ emphasize that care has to be exercised when interpreting long-pulse radar data, as reflector characteristics and mechanisms could change within the pulse length without resulting in obvious changes in the reflection magnitude or depth. The variation of reflection magnitude of the reflector from COF suggests that fabric changes are less continuous than conductivity characteristics, as they are susceptible to variations of internal stresses, e.g. between upstream and downstream sides of subglacial bedrock variations.

\subsection{Reflectors from COF - frequent or uncommon?}

Whereas certain flow regimes are necessary for sharp transitions in COF to occur, changes in conductivity are not constrained by comparable ice-dynamic conditions. We therefore expect that continuous internal reflectors from COF do occur less often than reflectors from conductivity. It is commonly expected that changes in conductivity cause isotropic reflectors. Reflections from $\mathrm{COF}$, instead, can be anisotropic or isotropic. Anisotropic reflections only occur if the underlying change in COF patterns is also anisotropic in the horizontal plane. This is usually the case in flow regimes with significant lateral strain (e.g. Fujita et al., 2003), as is the case at the EDML drilling site. In contrast, analyses at Dome Fuji down to a depth of $2500 \mathrm{~m}$ (about $83 \%$ ice thickness) by Fujita et al. (2006) indicate that isotropic reflections with birefringent effects prevail. They explain this by the horizontally-symmetric pattern in COF in the same depth range, caused by symmetric divide flow at the dome. Any potential reflections from changes in COF therefore also show an isotropic pattern at Dome Fuji.

Observations of either type of reflector, conductivity- or COF-based, are likely biased, as data acquisition required for ice-core analyses are more intensive for detection of COF-type reflectors. The same holds true for the type of RES measurements required to indirectly identify the reflector origin from RES measurements alone. Reflections from conductivity are independent of polarisation plane. Reflectors from COF can only be identified by azimuthdependent polarisation measurements at a single location in the case of anisotropic reflections, or a combination with multi-frequency measurements in the case of isotropic reflections. As azimuths-dependent RES measurements require more time than simple straight RES profiles (especially if only airborne systems are available), they are carried out less often.

To date, the reflector discussed here is the only one at EDML that can be clearly attributed to changes in COF. In contrast, 13 strong internal reflectors were identified to originate from changes in conductivity at the same location (Eisen et al., 2006). However, with more COF data emerging, it might be possible that some reflectors appear to be of a mixed type, with their origin being partly from changes in COF, partly from conductivity. For instance, the reflector only present in the 600-ns pulse data at 1850 -m depth could be caused by the series of conductivity peaks (Fig. 3), but it could also stem from slight changes in COF, or both. Further refinement of the COF data in at least $10-\mathrm{m}$ resolution is necessary to answer this question conclusively.

Some of the few ice cores from deep-drilling sites currently available display transitions from COF patterns which are anisotropic in the horizontal plane (e.g. girdle-type), to highly concentrated single-maximum fabrics in the lower part of the ice column, as at NorthGRIP (Wang et al., 2002) or Vostok (Obbard and Baker, 2007). However, only for some of the deep-drilling ice cores are profiles of COF available in high enough resolution to search for potential reflectors from COF directly. Another promising site to identify reflections from COF is the Siple Dome. The COF data, measured in 20-m intervals, show a sharp transition from an elongated to a highly concentrated fabric between 685 and 700-m depth, with the largest eigenvalue of the COF distribution changing from 0.75 to 0.95 (DiPrinzio et al., 2005, Fig. 3). RES data from the Siple Dome (e.g. Gades et al., 2000, Fig. 3) indicate a strong continuous internal reflector at about the same depth (based on the travel time depth conversion of Gades et al., 2000). In analogy to the findings developed here, this reflector thus bears the potential to stem from an abrupt change in COF.

Ice-core deep drillings are mostly carried out at such divide or dome locations as Dome Fuji or Siple Dome. Paleoclimatic interpretation of ice-core records usually incorporates ice-flow modeling. Recent modeling studies of ice flow at Siple Dome emphasize the significance of changes in COF for ice flow. Pettit et al. (2007) implemented fabric properties from borehole measurements in an anisotropic nonlinear flow law as part of an ice-sheet flow model. They found that the band of highly vertically concentrated COF in the lower $20-30 \%$ of the ice column causes concentrated bedparallel shearing $300 \mathrm{~m}$ above the bed. Moreover, compared to an isotropic fabric, the anisotropy considerably increases the size of the isochrone arch underneath the Siple Dome divide, and thus strongly influences the age-depth distribution. Pettit et al. (2007) assumed that the COF-profile is only a function of normalized depth with respect to ice thickness. The method presented here to identify continuous reflectors from changes in COF provides a way to improve this assumption. If a reflector is identified to originate from changes in $\mathrm{COF}$, its true depth tracked in RES profiles could be used as a proxy for the spatial distribution of the change in $\mathrm{COF}$, instead of extrapolating its normalized depth as observed in boreholes or ice cores. 


\section{Conclusions}

The combination of ice-core profiles of COF and the conductivity with RES data of different pulse lengths in our approach identifies the origin of a defined continuous internal reflector as a sharp transition in COF, from a girdle-type to a single-maximum fabric over just a few tens of meters. This finding goes beyond the previous analyses, which related RES signals to flow-induced COF, but did not provide a direct comparison of continuous internal layers in RES data with nearby in-situ data of COF. We showed that, even at short pulse lengths, the width of the COF-related reflector is comparable to reflectors stemming from conductivity peaks, limited by the RES pulse length. The spatial variation of the reflector from $\mathrm{COF}$ in depth is parallel to other internal reflectors, but less continuous. However, we emphasize that we have no evidence whether the reflector from $\mathrm{COF}$ is an isochrone or not. These observations imply that care has to be taken when correlating ice-core data with internal reflectors, if only single-frequency single-polarisation RES data are available, as these cannot pin down the reflector origin unambiguously.

The identification of a single change in $\mathrm{COF}$ as the origin of a strong individual internal reflector opens new possibilities for understanding and modeling ice dynamics. The fabric properties can be extrapolated along the reflector, as long as it is continuously trackable in the RES profile. Determination of the internal distribution of stresses can thus go beyond the point information provided by ice cores. Ice-dynamic models can utilize the reflector as an ancillary condition. As the rheology of a girdle-type fabric differs from those of a single-maximum fabric, the reflector indicates a boundary of different ice-flow properties above and below the reflector within the ice sheet. A remaining challenge lies in exclusively using remote-sensing data, like RES or seismics, to discriminate physical properties from the ice sheet's surface, without requiring in-situ information from ice cores. By providing characteristic observations of the forward relation of changes in COF and reflectivity, this study provides another step towards solving the inverse problem, which should eventually be used to determine the continuous profiles of vertical and lateral physical properties within the ice sheet.

Acknowledgements. This work is a contribution to the European Project for Ice Coring in Antarctica (EPICA), a joint European Science Foundation/European Commission scientific programme, funded by the EU and by national contributions from Belgium, Denmark, France, Germany, Italy, the Netherlands, Norway, Sweden, Switzerland and the United Kingdom. The main logistic support was provided by IPEV and PNRA (at Dome C) and AWI (at Dronning Maud Land). This is EPICA publication no. 186. Preparation of this work was supported by an "Emmy Noether"-scholarship of the Deutsche Forschungsgemeinschaft grant EI 672/1 to O. Eisen.

Edited by: J. L. Bamber

\section{References}

Bogorodsky, V. V., Bentley, C. R., and Gudmandsen, P. E.: Radioglaciology, D. Reidel Publishing Company, Dordrecht, Holland, 1985.

DiPrinzio, C. L., Wilen, L. A., Alley, R. B., Fitzpatrick, J. J., Spencer, M. K., and Gow, A. J.: Fabric and texture at Siple Dome, Antarctica, J. Glaciol., 51, 281-290, 2005.

Doake, C. S. M., Corr, H. J. F., and Jenkins, A.: Polarization of radio waves transmitted through Antarctic ice shelves, AG, 34, 165-170, 2002.

Dowdeswell, J. A. and Evans, S.: Investigations of the form and flow of ice sheets and glaciers using radio-echo sounding, Rep. Prog. Phys., 67, 1821-1861, 2004.

Durand, G., Gillet-Chaulet, F., Svensson, A., Gagliardini, O., Kipfstuhl, S., Meyssonnier, J., Parrenin, F., Duval, P., and DahlJensen, D.: Change of the ice rheology with climatic transitions - implications for ice flow modelling and dating of the EPICA Dome C core, Clim. Past, 3, 155-167, 2007, http://www.clim-past.net/3/155/2007/.

Eisen, O., Wilhelms, F., Steinhage, D., and Schwander, J.: Improved method to determine RES-reflector depths from ice-core profiles of permittivity and conductivity, J. Glaciol., 52, 299310, 2006.

Fujita, S., Maeno, H., Uratsuka, S., Furukawa, T., Mae, S., Fujii, Y., and Watanabe, O.: Nature of radio echo layering in the Antarctic ice sheet detected by a two-frequency experiment, J. Geophys. Res., 104, 13 013-13 024, 1999.

Fujita, S., Matsuoka, T., Ishida, T., Matsuoka, K., and Mae, S.: A summary of the complex dielectric permittivity of ice in the megahertz range and its application for radar sounding of polar ice sheets, in: The Physics of Ice Core Records, edited by: Hondoh, T., pp. 185-212, Hokkaido University Press, 1 edn., 2000.

Fujita, S., Matsuoka, K., Maeno, H., and Furukawa, T.: Scattering of VHF radio waves from within an ice sheet containing the vertical-girdle-type ice fabric and anisotropic reflection boundaries, Ann. Glaciol., 37, 305-316, 2003.

Fujita, S., Maeno, H., and Matsuoka, K.: Radio-wave depolarization and scattering within ice sheets: a matrix-based model to link radar and ice-core measurements and its application, J. Glaciol., 52, 407-424, 2006.

Gades, A., Raymond, C., Conway, H., and Jacobel, R.: Bed properties of Siple Dome and adjacent ice streams, West Antarctica, inferred from radio-echo sounding measurements, J. Glaciol., 46, 88-94, 2000.

Hargreaves, N. D.: The radio-frequency birefringence of polar ice, J. Glaciol., 21, 301-313, 1978.

Harrison, C. H.: Radio echo sounding of horizontal layers in ice, J. Glaciol., 12, 383-397, 1973.

ISMASS Committee: Recommendations for the collection and synthetis of Antarctic Ice Sheet mass balance data, Global Planet. Change, 42, 1-15, 2004.

Liu, H., Jezek, K., Li, B., and Zhao, Z.: Radarsat Antarctic Mapping Project digital elevation model version 2, Digital media, National Snow and Ice Data Center, Boulder, CO, USA, 2001.

Marshall, S. J.: Recent advances in understanding ice sheet dynamics, E. Plan Sci. Lett., 240, 191-204, doi:10.1016/j.epsl.2005.08.016, 2005.

Matsuoka, K., Furukawa, T., Fujita, S., Maeno, H., Uratsuka, S., Naruse, R., and Watanabe, O.: Crystal orientation fabrics within 
the Antarctic ice sheet revealed by a multipolarization plane and dual-frequency radar survey, J. Geophys. Res., 108(B10), 2499, doi:10.1029/2003JB002425, 2003.

Matsuoka, K., Uratsuka, S., Fujita, S., and Nishio, F.: Ice-flow induced scattering zone within the Antarctic ice sheet revealed by high-frequency airborne radar, J. Glaciol., 50, 382-388, 2004.

Nixdorf, U., Steinhage, D., Meyer, U., Hempel, L., Jenett, M., Wachs, P., and Miller, H.: The newly developed airborne RESsystem of the AWI as a glaciological tool, Ann. Glaciol., 29, 231-238, 1999.

Obbard, R. and Baker, I.: The microstructure of meteoric ice from Vostok, Antarctica, J. Glaciol., 53, 41-62, 2007.

Paren, J. G.: PRC at a dielectric interface, J. Glaciol., 27, 203-204, 1981.

Paren, J. G. and Robin, G. de Q.: Internal reflections in polar ice sheets, J. Glaciol., 14, 251-259, 1975.

Paterson, W. S. B.: The physics of glaciers, 3rd edition, Oxford, New York, Tokyo, Pergamon, ix, 480 pp., ISBN 0-08037944 3, 1994.

Petrenko, V. F. and Whitworth, R. W.: Physics of Ice, Oxford University Press, 1999.
Pettit, E. C., Thorsteinsson, T., Jacobson, H. P., and Waddington, E. D.: The role of crystal fabric in flow near an ice divide, J. Glaciol., 53, 277-288, 2007.

Robin, G. de Q., Evans, S., and Bailey, J. T.: Interpretation of radio echo sounding in polar ice sheets, in: Philosophical Transactions of the Royal Society of London, vol. 146 of A, pp. 437-505, Royal Society of London, 1969.

Wallbrecher, E.: Methoden zum quantitativen Vergleich von Regelungsgraden und Formen strukturgeologischer Datenmengen mit Hilfe von Vektorstatistik und Eigenwertanalyse, N. JB. Geol. Paläontol. Abh., 159, 113-149, 1979.

Wang, Y., Thorsteinsson, T., Kipfstuhl, J., Miller, H., Dahl-Jensen, D., and Shoji, H.: A vertical girdle fabric in the NorthGRIP deep ice core, North Greenland, Ann. Glaciol., 35, 515-520, 2002.

Wesche, C., Eisen, O., Oerter, H., Schulte, D., and Steinhage, D.: Surface topography and ice flow in the vicinity of EDML deepdrilling site, J. Glaciol., 53(182), 442-448, 2007.

Wilhelms, F.: Explaining the dielectric properties of firn as a density and conductivity mixed permittivity (DECOMP), Geophys. Res. Lett., 32, L16 501, doi:10.1029/2005GL022808, 2005.

Wilson, J., Russell-Head, D. S., and Sim, H. M.: The application of an automated fabric analyzer systm to the textural evolution of folded ice layers in shear zones, Ann. Glaciol., 37, 7-17, 2003. 